\title{
Screw dislocation induced phonon transport suppression in SiGe superlattices
}

\author{
Song Hu, ${ }^{1}$ Hongping Zhang, ${ }^{2}$ Shiyun Xiong, ${ }^{3}$ Honggang Zhang, ${ }^{1}$ Hongyan Wang, ${ }^{1}$ Yuanzheng Chen, ${ }^{1}$ \\ Sebastian Volz, ${ }^{4,5}$ and Yuxiang $\mathrm{Ni}$ (i) ${ }^{1, *}$ \\ ${ }^{1}$ School of Physical Science and Technology, Key Laboratory of Advanced Technologies of Materials, Ministry of Education of China, \\ Southwest Jiaotong University, 610031 Chengdu, People's Republic of China \\ ${ }^{2}$ Engineering Research Center of Biomass Materials, Ministry of Education, School of Materials Science and Engineering, Southwest \\ University of Science and Technology, 621010 Mianyang, People's Republic of China \\ ${ }^{3}$ Functional Nano and Soft Materials Laboratory (FUNSOM) and Collaborative Innovation Center of Suzhou Nano Science and Technology, \\ Soochow University, 215123 Suzhou, People's Republic of China \\ ${ }^{4}$ Laboratoire EM2C, CNRS, CentraleSupélec, Université Paris-Saclay, Grande Voie des Vignes, 92295 Châtenay-Malabry Cedex, France \\ ${ }^{5}$ LIMMS/CNRS-IIS(UMI2820)Institute of Industrial Science, University of Tokyo 4-6-1 Komaba, Meguro-ku, 153-8505 Tokyo, Japan
}

(Received 23 April 2019; published 26 August 2019)

\begin{abstract}
Screw dislocations are known to impede the thermal transport of homogeneous nanowires by reducing the phonon relaxation time without affecting the phonon group velocity. By using molecular dynamics simulations in this study, we show that the impact of screw dislocation on the thermal conductivity of the SiGe superlattice nanowires depends on the period length. The analysis of phonon transmission spectra and phonon mean free paths indicate that strong phonon-screw dislocation scatterings occur for phonons in the frequency range of 3-8 THz. The screw dislocations change the phonon scattering mechanisms, which is the main cause of the thermal conductivity reduction. Contrary to the case of homogeneous nanowires, a sizable decrease in the phonon group velocity is found in superlattices with screw dislocations. This phenomenon is attributed to the larger number of Si-Ge bonds in the vicinity of the interface due to the slipping of the atomic planes. In contrast to the decreased thermal conductivity, the phonon propagation in the interface region of the nanowires is enhanced by screw dislocations. Our findings provide critical insights into the understanding of dislocation-heat transfer relationship in materials, especially in heterostructures where interfaces are vital for thermal transport.
\end{abstract}

DOI: $10.1103 /$ PhysRevB.100.075432

\section{INTRODUCTION}

Dislocations are known to influence the formation and migration of point defects in crystalline materials, which has a profound impact on the physics of substances [1,2], including thermal properties [3]. Understanding and characterizing the screw dislocation (SD) effects on the thermal properties of bulk and nanomaterials has long been a challenge. Klemens first treated the static phonon-dislocation scattering using perturbation theory [4,5], which was recently found to be insufficient in describing the dislocation impacts on the thermal properties, from both theoretical [6] and experimental perspectives [1].

In recent years, Dumitrică et al. investigated the relationship between screw dislocation and the electrical and thermal properties of a series of materials, including $\mathrm{ZnO}$ and $\mathrm{Si}$ nanowires and nanotubes, $\mathrm{SiC}, \mathrm{PbSe}$, and $\mathrm{SiGe}$ nanowires [6-11]. The observed narrower bandgaps and lower thermal conductivity in nanowires induced by screw dislocations bring new perspectives to the nanoscale design of electronic and thermoelectric materials. For thermal transport, SD was identified to be a source of anharmonic phonon-phonon scattering and reduces the relaxation time of longitudinal acoustic phonons [6]. Termentzidis et al. [12] studied the impact of

*yuxiang.ni@swjtu.edu.cn
SDs on the thermal conductivity of individual nanowires and bulk $\mathrm{GaN}$, and found that the interaction between phonons and the core of dislocations and their strain field leads to the decrease of the thermal conductivity. Very recently, Sun et al. [1] measured anisotropic heat transport in single-crystal indium nitride films, which is induced by the oriented edge-type and screw-type dislocations. These studies have significantly advanced the knowledge about SDs and how they impact thermal transport in crystals and nanostructures.

However, the phonon-dislocation interactions observed in homogeneous materials may behave differently in heterostructures, where phonon-interface scattering plays an important role in heat transfer. Therefore, it remains an open question whether screw dislocations can result in a major suppression of thermal conductivity not only in homogeneous materials, but also in heterostructures with multiple interfaces such as superlattices (SLs). The screw dislocations were previously observed experimentally not only in homogeneous materials [13-17] but also in nanocrystals superlattices $[18,19]$. In this regard, it is imperative to achieve a direct and accurate understanding of the effect of SDs on the thermal transport of superlattices.

Due to their important applications in microelectronics and thermoelectrics, SiGe SLs and SL nanowires (NWs) have been studied extensively in terms of thermal transport [20-31]. It also serves as an ideal model to introduce screw dislocations due to the small lattice mismatch between $\mathrm{Si}$ 


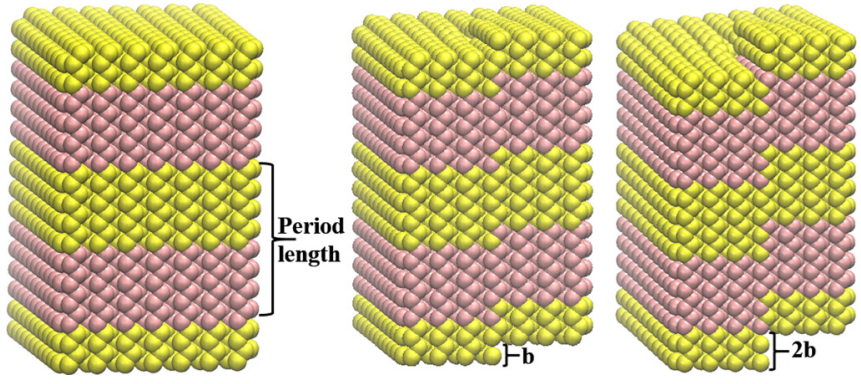

FIG. 1. Pristine (left), $1 b$ (center), and $2 b$ (right) screw dislocated $\mathrm{Si} / \mathrm{Ge} \mathrm{SL}$ NWs. The cross-sectional area is $4.3 \times 3.8 \mathrm{~nm}^{2}$. The length of the nanowires is $96.0 \mathrm{~nm}$.

and Ge. In this study, by using molecular dynamics (MD) simulations, we investigate the Burgers vector and periodlength-dependent thermal transport in SiGe SL NWs. We aim to not only uncover the impact of SDs on the thermal conductivity, but also reveal how SDs affect the interfacial thermal resistance in SLs. Phonon analyses including the calculations of transmission spectra, dispersion relation, and group velocity were performed to better understand the physical mechanisms of the obtained results.

The organization of this paper is as follows. In Sec. II, we describe the atomic models of SiGe SL NWs in the calculations, and the methods used for the thermal transport calculations and phonon analyses. In Sec. III, we show the results of the period-length- and screw-dislocation-dependent thermal conductivity, together with the related phonon analyses. In Sec. IV, we show the impact of SDs on the interface thermal resistance of SiGe SL NWs. Finally, the main findings of this work are summarized in Sec. V.

\section{METHOD}

\section{A. Models}

We start with $4.3 \times 3.8 \times 96.0 \mathrm{~nm}^{3}$ pristine SiGe SL NWs with period lengths (PLs) ranging from 0.75 to $48 \mathrm{~nm}$. Normal to the $\mathrm{Si} / \mathrm{Ge}$ interface is the [110] crystal direction, which was reported to be stable in disorder and surface atom reconstruction [32]. The lattice constant of $\mathrm{Ge}$ is assumed to be the same as that of $\mathrm{Si}(a=5.43 \AA)$ in our simulations due to their relatively small lattice mismatch, as was done in Ref. [26]. We put the periodic boundary condition only in the $z$ direction, which was along the nanowires. Screw dislocations were introduced to the pristine SL structures with the axis (along the $z$ direction) located at the center, see Fig. 1. We considered a minimal Burgers vector of magnitude $1 b$ and $2 b$, where $b=3.84 \AA$.
Models shown in Fig. 2 were used in the nonequilibrium MD (NEMD) simulations for the phonon transmission spectra calculations. Because of the multiple interfaces in the SLs, it is difficult to obtain the thermal conductivity $(\kappa)$ from the direct method in NEMD. We thus resort to equilibrium MD (EMD) simulations and the Green-Kubo method.

\section{B. Thermal conductivity calculation of SiGe SL NWs with screw dislocation}

The calculations were performed with the equilibrium molecular dynamics (EMD) simulation by using the Lammps package [33]. Tersoff potential was used to describe the atomic interactions [34,35], which has widely been used to study the thermal conductivities and interface thermal resistance of SiGe systems $[25,26,30]$ The initial structures were relaxed by using the conjugate gradient energy minimization algorithm. The NW structures were then evolved for $200 \mathrm{ps}$ in the canonical ensemble (NVT) in order to impose a temperature of $300 \mathrm{~K}$, and next in the microcanonical ensemble (NVE) for another $200 \mathrm{ps}$ to achieve thermal equilibrium. The MD time step was set to 0.2 fs to avoid the impact of energy drift on the calculated results. Finally, MD runs with lengths equal to $200 \mathrm{ps}$ were carried out to calculate the thermal conductivity along the $z$ axis $\left(\kappa_{z}\right) . \kappa_{z}$ was computed based on the instantaneous $z$ component of the microscopic heat flux $j_{z}(t)$ and the Green-Kubo formula [36]

$$
\kappa_{z}=\frac{V}{k_{B} T^{2}} \int_{0}^{+\infty}\left\langle j_{z}(t) j_{z}(0)\right\rangle d t .
$$

In these expressions, $k_{B}$ is the Boltzmann constant, $T$ is the temperature, and $V$ is the system volume. The angular brackets denote the ensemble average, performed here over the microstates of $20 \mathrm{MD}$ measurement runs. To ensure the obtained results and the main conclusions are independent of the modeling parameters, we also calculated the thermal conductivity from the Stillinger-Weber interatomic potential, and the results are given in the Supplemental Material [37]. Also shown in the Supplemental Material is the running thermal conductivity for SiGe SL NW as a function of correlation time.

\section{Phonon transmission, mean free path, and group velocity calculations}

To identify the mechanism underling the SD effect on the phonon transport of SL NWs, we calculated the phonon transmission spectra $T(\omega)$ of the SiGe SL NWs from nonequilibrium molecular dynamics (NEMD). $T(\omega)$ was calculated based on the method developed by Sääskilahti et al. [38,39].

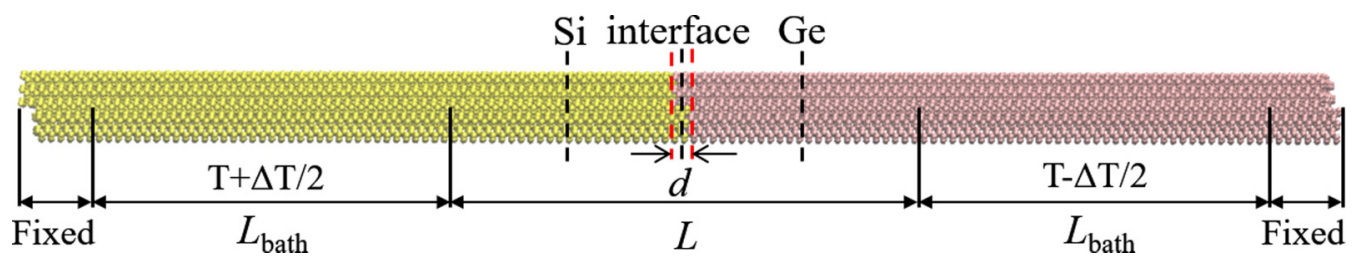

FIG. 2. Model used in the phonon transmission calculations. The black dashed lines indicate the three different locations along the wire for the phonon transmissioncalculations. 
The frequency resolved heat flux between atom $i$ and $j$ can be expressed as

$$
q_{i \rightarrow j} \approx-\frac{2}{t_{\text {simu }} w} \sum_{\alpha, \beta \in\{x, y, z\}} \operatorname{Im}\left\langle\widehat{v}_{i}^{\alpha}(w) * K_{i j}^{\alpha \beta} \widehat{v}_{j}^{\beta}(w)\right\rangle,
$$

where $t_{\text {simu }}$ is the simulation time and $w$ is the frequency. $\widehat{v}_{i}^{\alpha}(w)$ and $\widehat{v}_{j}^{\beta}(w)$ are the Fourier transformed atomic velocities of atom $i$ in the $\alpha$ direction and atom $j$ in the $\beta$ direction. For calculating the flux spectra, the force constant matrix is calculated with the finite displacement method by LAMMPS; i.e., after relaxing the structures to the potential minimum, atom $i$ is moved in the directions $\pm x, \pm y$, and $\pm z$ with a small value $(\Delta=0.01 \AA)$. After each displacement, the forces $F$ of each atom are calculated. As a result, the element of the force constant matrix can be evaluated as

$$
K_{i j}^{\alpha \beta}=\frac{F_{j}^{\beta-}-F_{j}^{\beta+}}{2 \Delta} .
$$

Here $F_{j}^{\beta-}$ and $F_{j}^{\beta+}$ denote the force on atom $j$ in $\beta$ direction when atom $i$ is displace to $-\alpha$ and $+\alpha$ directions, respectively.

The heat current across any interface separating adjacent atom sets $L$ and $R$ can be obtained by summing over atoms in each set:

$$
q(\omega)=\sum_{i \in L} \sum_{j \in R} q_{i \rightarrow j}(\omega),
$$

then the phonon transmission function can be defined according to the spectral heat current as

$$
\operatorname{Trans}(\omega)=\frac{q(\omega)}{k_{B} \Delta T},
$$

where $\Delta T$ is the temperature difference of the two thermal baths in NEMD simulations. Figure 2 illustrates the model used in the phonon transmission calculations. The atoms at the two ends of the structures shown in Fig. 2 are fixed. The atoms located within the distance $L_{\text {bath }}=20 \mathrm{~nm}$ from left and right adjoined to fixed areas are coupled to hot and cold Langevin heat baths at temperatures $T+\Delta T / 2$ and $T-$ $\Delta T / 2$, respectively. The section between the thermal baths is $12.2 \mathrm{~nm}$ long. The MD time step was set to $0.5 \mathrm{fs}$, and the coupling time constant of the Langevin thermostat was chosen as $\tau_{\text {time }}=1 \mathrm{ps}$. The mean thermal bath temperature was fixed as $T=300 \mathrm{~K}$, and $\Delta T$ was set as $60 \mathrm{~K}$. We investigated the phonon transmission at three different locations along the wire, respectively, in $\mathrm{Si}, \mathrm{Ge}$, and at the interface, as indicated by the dashed lines in Fig. 2.

From the phonon transmission spectra, we evaluated the frequency resolved phonon mean free paths (MFPs) from the following equation $[40,41]$ :

$$
\operatorname{Trans}(\omega)=\frac{M(\omega)}{1+\frac{L}{\Lambda(\omega)}},
$$

where the $M(\omega)$ is the number of phonon modes at a given frequency $\omega, L$ is the system length between the thermal baths as demonstrated in Fig. 2, and $\Lambda(w)$ is the effective phonon MFP at frequency $\omega$. The phonon dispersion relations and the group velocity of SiGe SL NWs were obtained based on harmonic lattice dynamics $[42,43]$.
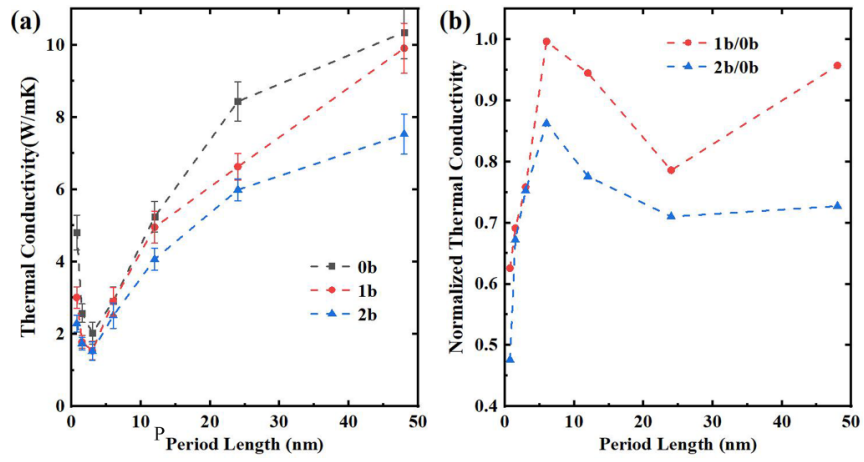

FIG. 3. (a) Thermal conductivity of the pristine SiGe SL NWs $(0 b)$ and the screw-dislocated ones $(1 b$ and $2 b)$ with different period lengths. (b) Thermal conductivity of the screw-dislocated SiGe SL NWs normalized by that of the pristine structures.

\section{RESULTS AND DISCUSSION}

\section{A. Thermal conductivity of SiGe SL NWs with screw dislocation}

Figure 3(a) shows the calculated thermal conductivity of $\mathrm{SiGe}$ SL NWs at $300 \mathrm{~K}$ for both pristine and screw-dislocated structures and different period lengths. A minimum thermal conductivity is obtained for all the structures at short periods, indicating the existence of coherent phonons in both pristine and screw-dislocated SLs. It is a result of the interplay between the decrease in group velocity and the increase in phonon lifetimes with increasing SL period [44] (in the next section we will show that the group velocity first decreases with period length (from $\mathrm{PL}=0.75 \mathrm{~nm}$ to $\mathrm{PL}=1.5 \mathrm{~nm}$ ) and then remain unchanged). At the smallest period length $(0.75 \mathrm{~nm})$, there is a distinct reduction in $\kappa$ of the structures with SDs, and $\kappa$ decreases with the Burgers vector $b$. For the pristine SL NWs, the lower-frequency phonons that contribute to the thermal conductivity were mostly coherent during their transport through the SL structures until they were scattered at the NW boundaries. As the coherent length is limited, when SDs are involved, the dislocation line, which exists throughout the entire wire, provides additional scattering sites for the coherent phonons. This led to a reduction in their heat-carrying ability and caused an overall decrease in $\kappa$. For the samples with period lengths of 1.5 and $3.1 \mathrm{~nm}$, the discrepancy in $\kappa$ between the pristine structures and the dislocated ones vanished, as the data are within the error bars. In this period length region, the phonon coherent length is comparable with period length, and the coherent phonons are scattered by the interfaces. In this case, the decrease in $\kappa$ is a combined effect of interface scattering and phonon-dislocation line scattering, and their competition weakens the SD effect in $\kappa$ suppression. As the period length further increases, the interface density along the NW gets smaller, and the phonon-SD scattering becomes predominant. Sizable reduction in $\kappa$ can thus be seen in the structures with SDs, compared with the thermal conductivity of the pristine structures. The SD effect on the thermal conductivity is also found for NWs with larger cross-sectional area [37]. To better demonstrate the $\kappa$ reduction, Fig. 3(b) shows the thermal conductivity of the screw-dislocated SiGe SL NWs normalized by that of the pristine structures. 

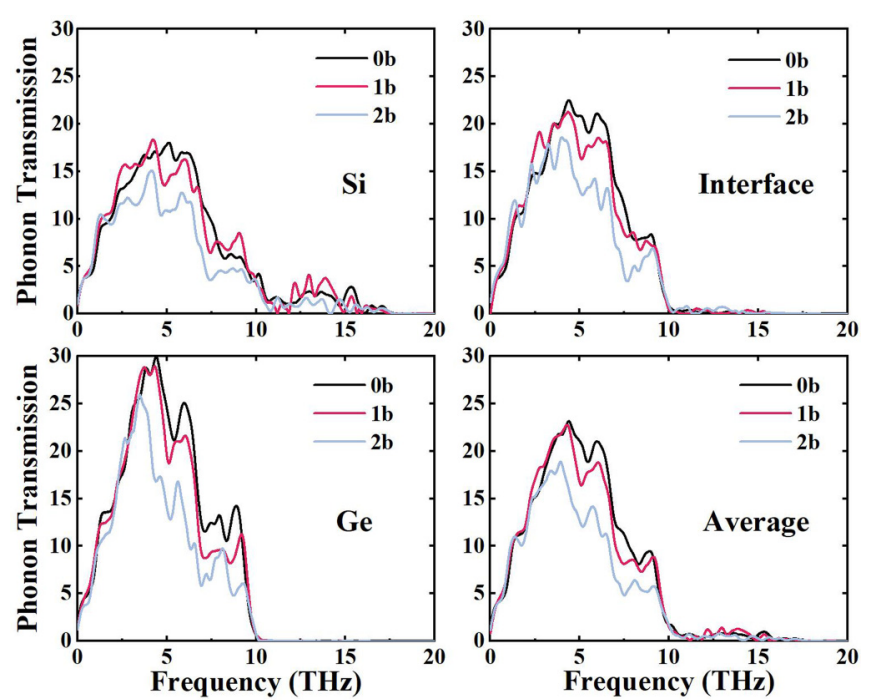

FIG. 4. Phonon transmission spectra of SiGe SL NWs in the pure $\mathrm{Si}$, the interface, and the pure $\mathrm{Ge}$ sections. The averaged spectrum for the three locations is also shown.

\section{B. Phonon analysis of SiGe SL NWs with screw dislocation}

\section{Phonon transmission and mean free paths}

Figure 4 depicts the Burgers-vector-dependent phonon transmission spectra at $300 \mathrm{~K}$. Trans $(\omega)$ averaged from the three different locations is also shown. In general, Trans $(\omega)$ for the three different locations exhibits similar behaviors. The transmission of the low-frequency phonons (below $3 \mathrm{THz}$ ) is not sensitive to the screw dislocations due to their longwavelength nature. However, the spectra decreases with $b$ typically in the frequency range $3-8 \mathrm{THz}$, indicating the strong phonon-SD scattering. Screw dislocation represents a significant source of anharmonic phonon-phonon scattering, and the resulting lower phonon transmission is in accordance with the $b$-dependent thermal conductivity shown in Fig. 3.

By using Eq. (5), we calculated the frequency resolved phonon MFP evaluated at $\mathrm{Si}, \mathrm{Ge}$, and the interface, as shown in Fig. 5. As revealed in the figure, SD decreases the phonon MFP at the frequency range 3-8 THz. The decrease in MFP stems from the shorter phonon relaxation time with increasing Burgers vector, as reported for $\mathrm{SiC}$ NWs in our previous work [6].

To further identify the phonon scattering mechanisms, we fitted the MFP as a function of the phonon frequency $\omega$, and the results are demonstrated in Fig. 6. For the phonons in the frequency range of $2.5-6 \mathrm{THz}$, screw dislocation does not show obvious impact on the fitting behaviors. The MFP in Ge sections is proportional to $\omega^{-2}$, a consequence of phononphonon scattering. For the phonons with the frequency between 1 and $2 \mathrm{THz}$, the fitting behavior is significantly affected by SDs. The MFPs of the phonons in this frequency range in the pristine $\mathrm{Si}$ and $\mathrm{Ge}$ sections are proportional to $\omega^{-2.6}$ and $\omega^{-3.1}$, respectively. With the appearance of SDs, the scattering obeys the Rayleigh law, and the MFPs in both sections changed to be proportional to $\omega^{-4}$, which corresponds to the phonon-dislocation scatterings [45]. The above analysis of
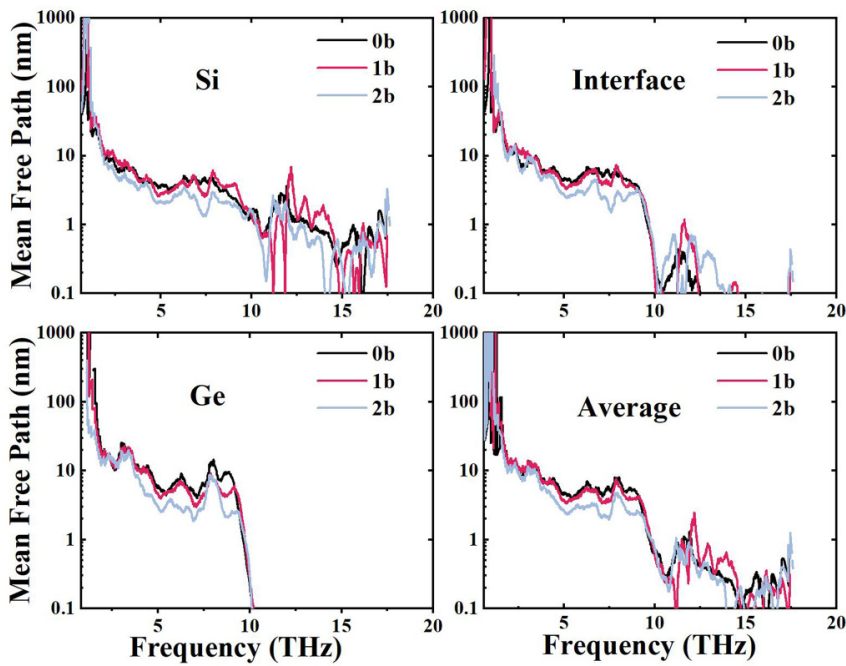

FIG. 5. Frequency resolved phonon mean free path in the pure $\mathrm{Si}$, the interface, and the pure Ge sections. The averaged spectrum for the three locations is also shown.

$\Lambda(w)$ fitting behaviors indicates that SDs change the phonon scattering mechanisms, which is the cause of the $\kappa$ reduction.

\section{Phonon group velocity in SiGe SL NWs}

Figure 7 depicts the phonon group velocity $V_{g}$ of the pristine SiGe SL NWs and the screw-dislocated ones $(2 b)$ with different period lengths. The lattice distortion by SDs shortens the phonon relaxation time, but rarely changes $V_{g}$ due to the unaltered force constants, as reported in the previous study for SiC NW [6]. Unexpectedly, a sizable decrease in

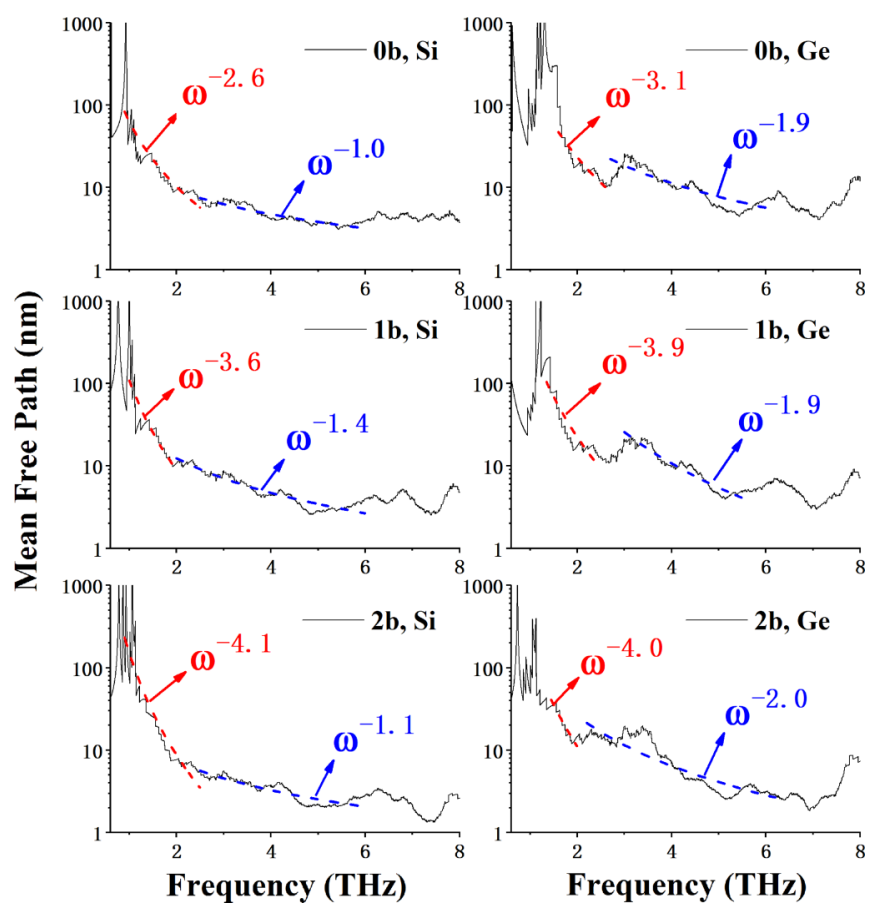

FIG. 6. Phonon mean free paths of the SiGe systems. The dashed lines indicate the fitting with the phonon frequency $\omega$. 


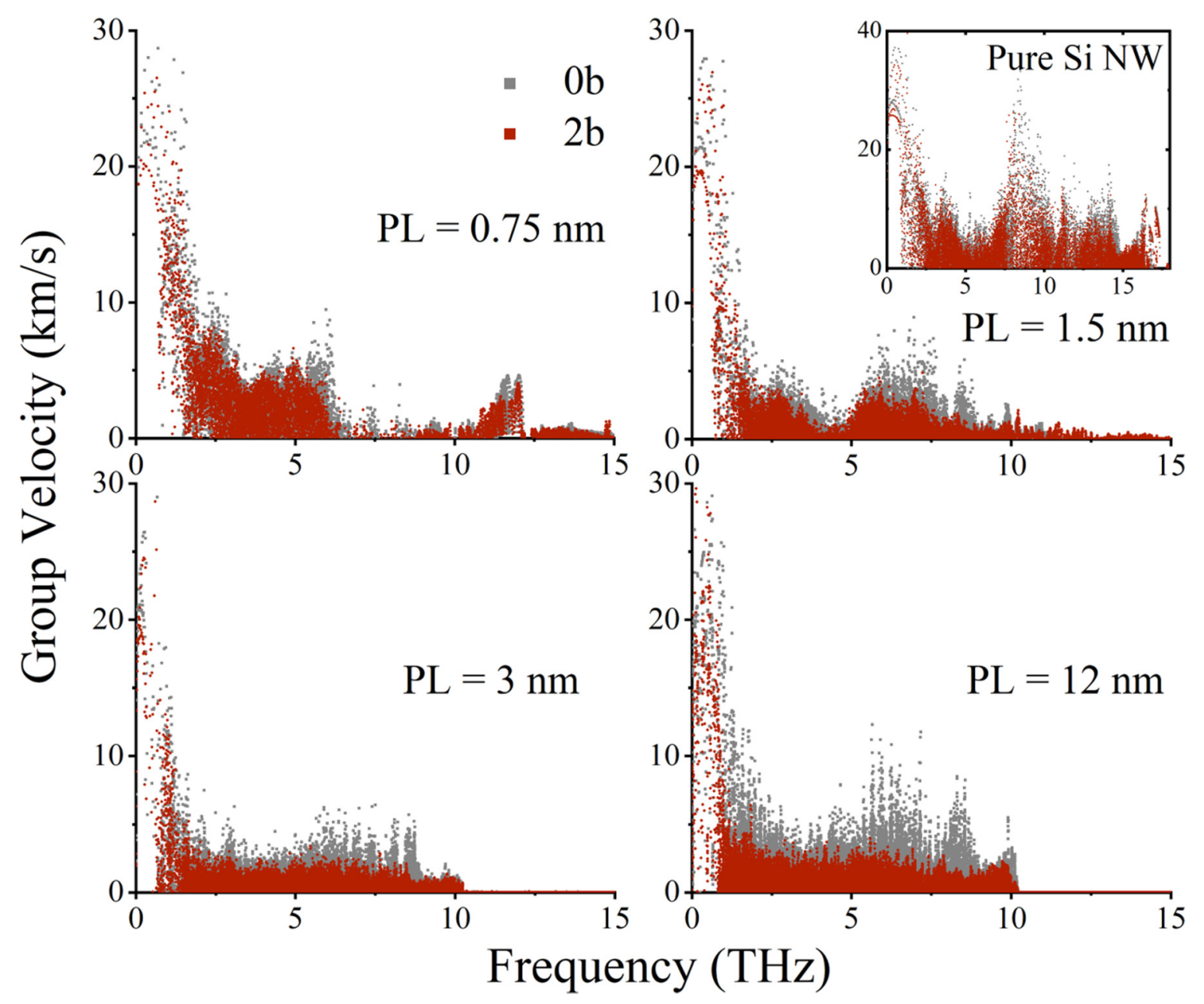

FIG. 7. Frequency resolved phonon group velocity $V_{g}$ of the pristine SiGe SL NWs $(0 b)$ and the screw dislocated ones $(2 b)$ with different period lengths. The insert shows the $V_{g}$ of a pure Si NW with the same cross-sectional area and length.

$V_{g}$ is found for the dislocated SLs with PL $=1.5,3$, and $12 \mathrm{~nm}$, compared with the pristine structures. To elucidate this observation, account has to be taken of the fact that the number of Si-Ge bonds $\left(N_{\mathrm{Si}-\mathrm{Ge}}\right)$ in the vicinity of the interface is increased due to the slipping of the atomic planes in the screw dislocated structures. Table I summarizes the $N_{\mathrm{Si} \text {-Ge }}$ in the vicinity of the interface for $0 b, 1 b$, and $2 b$ structures, with a cross-sectional area of $4.3 \times 3.8 \mathrm{~nm}^{2}$. An increase can be found in $N_{\mathrm{Si}-G e}$ in the dislocated structures, except for the one with the smallest period length $(\mathrm{PL}=0.75 \mathrm{~nm})$. A larger $N_{\mathrm{Si}-\mathrm{Ge}}$ indicates more phonon backscattering due to

TABLE I. Number of Si-Ge bonds in the vicinity of the interface for $0 b, 1 b$, and $2 b$ structures, with a cross-sectional area of $4.3 \times$ $3.8 \mathrm{~nm}^{2}$.

\begin{tabular}{llll}
\hline \hline Period length $(\mathrm{nm})$ & $0 b$ & $1 b$ & $2 b$ \\
\hline 0.75 & 68 & 68 & 67 \\
1.5 & 68 & 76 & 75 \\
3.0 & 68 & 76 & 81 \\
6.0 & 68 & 76 & 81 \\
12.0 & 68 & 76 & 81 \\
\hline \hline
\end{tabular}

the acoustic impedance mismatch, which is presumably the cause of the $V_{g}$ decrease in the dislocated structures. For the model with $\mathrm{PL}=0.75 \mathrm{~nm}, N_{\mathrm{Si}-\mathrm{Ge}}$ is not significantly changed since the Burgers vector is comparable with the thickness of $\mathrm{Si}$ (Ge) layers, and accordingly, there is no obvious change in $V_{g}$ against the Burgers vector. To further confirm our conjectures, we calculated $V_{g}$ of pure $\mathrm{Si} \mathrm{NW}$, and the results are shown in the insert of Fig. 7. In the pure $\mathrm{Si} \mathrm{NW}$ where there is only homoelemental ( $\mathrm{Si}-\mathrm{Si}$ ) bonds, $V_{g}$ is not sensitive to $\mathrm{SD}$, indicating that the decrease of $V_{g}$ is due to the acoustic impedance mismatch rather than the anharmonicity caused by SDs.

\section{Impact of SDs on the interface thermal resistance of SiGe SL NWs}

Different from the homogeneous materials, superlattice is a periodic structure of layers of different materials, which involves multiple interfaces. The slip of the atomic planes due to SDs changes the interface structure, which may affect the interface thermal resistance $\left(R_{\text {int }}\right)$. Unfortunately, it is unclear how SDs affect $R_{\text {int }}$ in superlattices. To make progress, we studied the Burgers-vector-dependent thermal resistance in 
TABLE II. Effective thermal resistance $\left(10^{-9} \mathrm{~m}^{2} \mathrm{~K} / \mathrm{W}\right)$ for the interface region of the SiGe SL NWs for pristine $(0 b)$ and screw dislocated structures.

\begin{tabular}{lccc}
\hline \hline Period length $(\mathrm{nm})$ & $0 b$ & $1 b$ & $2 b$ \\
\hline 48 & 1.50 & 1.49 & 1.34 \\
56 & 1.58 & 1.46 & 1.22 \\
\hline \hline
\end{tabular}

the vicinity of the interface in the SiGe SL NWs from NEMD simulations.

Since the interface is not neat for the systems with SDs, we define $R_{\text {eff }}$ as the effective thermal resistance for the interface region, which was treated as a series resistance that connects $\mathrm{Si}$ and Ge:

$$
R_{\mathrm{tot}}=l_{\mathrm{Si}} / \kappa_{\mathrm{Si}}+R_{\mathrm{eff}}+l_{\mathrm{Ge}} / \kappa_{\mathrm{Ge}},
$$

where $\kappa_{\mathrm{Si}}\left(\kappa_{\mathrm{Ge}}\right)$ and $l_{\mathrm{Si}}\left(l_{\mathrm{Ge}}\right)$ are the thermal conductivity and length of the corresponding $\mathrm{Si}(\mathrm{Ge})$ segment in the structure, respectively. Note that $\kappa_{\mathrm{Si}}$ and $\kappa_{\mathrm{Ge}}$ are model dependent. $l_{\mathrm{Si}}$ $\left(l_{\mathrm{Ge}}\right)$ is taken as $\left(L_{\mathrm{tot}}-d\right) / 2$ where $L_{\mathrm{tot}}$ is the system length between the thermal baths, and $d$ is the length of a short section that covers the interface region, chosen here as $0.94 \mathrm{~nm}$ (see the section between the red dashed lines of Fig. 2). The total resistance $R_{\text {tot }}$ is calculated by applying Fourier's law to the whole structure. The linear part of the temperature gradient was used to calculate the thermal conductivities of the $\mathrm{Si}$ and $\mathrm{Ge}$ sections. We studied the $R_{\text {eff }}$ of pristine SiGe NW SL as well as the ones with Burgers vectors equal to $1 b$ and $2 b$. We studied two period lengths which equal 36 and $46 \mathrm{~nm}$. The obtained results are summarized in Table II.

In contrast to the SD effect on the thermal conductivity, the phonon propagation in the interface region of the SiGe SL NWs is enhanced with SD. According to Table II, $R_{\text {eff }}$ is smaller in the models with SDs compared with that in the pristine ones, especially for $2 b$. However, the thermal conduction is not supposed to be enhanced since the SD-induced anharmonicity is adverse to the heat transport. To explain this unexpected phenomenon, one needs to look into the discrepancies that SDs bring to the interface structure. As shown in Fig. 1, SD leads to the slipping of atomic planes, which increases the area of Si-Ge contact by introducing additional interface parallel to the dislocation line. Based on the classical acoustic mismatch model (AMM), Merabia and Termentzidis [46] predicted that for phonons having a wavelength $\lambda$ smaller than the interfacial roughness parameter, the interfacial thermal conductance increases with the true interfacial area. In our case, SD causes an interface roughness $h$, which equals the magnitude of Burgers vector $\mathbf{b}$. When $h$ is small, the wavelengths of the phonons are in general larger than $h$ and they see the interface as a planar one: the transmitted heat flux is then controlled by the projected area. On the other hand, when the interface becomes rough due to SDs, most of the phonons have a wavelength smaller than the magnitude of the Burgers vector, in which case the phonons no longer feel the interface as planar, phonon scattering becomes completely incoherent, and the transmitted heat flux is controlled by the true surface area.

The increase of the phonon propagation in the vicinity of the interface may also be due to the inelastic phonon scatterings in the core region of SD. The lattice distortion in the core region creates additional phonon frequencies beyond the cutoff frequency [6], allowing for the phonon transmission above the phonon cutoff frequency of Ge. This assumption is corroborated by Fig. 4, where the interface transmission is found between $10-15 \mathrm{THz}$ for screw dislocated structures, which is beyond the cutoff frequency of $\mathrm{Ge}(\approx 10 \mathrm{THz})$. The interface conductance is believed to govern the thermal conductivity of SLs [28]; however, it is interesting to note that this phenomenon is no longer valid in SLs with SDs.

\section{CONCLUSIONS}

In conclusion, we investigated the thermal transport-screw dislocation relationship in SiGe SL NWs by molecular dynamics simulations. The coherent phonons are scattered by the SDs at small period lengths while they ignore the interfaces. As the period length becomes longer, the decrease in $\kappa$ is a combined effect of interface scattering and phonondislocation line scattering, and their competition weakens the SD effect in $\kappa$ suppression. As the period length further increases, the phonon-SD scattering becomes predominant. The calculated phonon transmission spectra and phonon mean free paths show that strong phonon-SD scattering happens for phonons in the frequency range 3-8 THz, and SDs change the phonon scattering mechanisms. Contrary to that in homogeneous nanowires, a sizable decrease in the phonon group velocity is found in superlattices with screw dislocations. This phenomenon is attributed to the larger number of Si-Ge bonds in the vicinity of the interface due to the slipping of the atomic planes. In contrast to the SD effect on the thermal conductivity, the phonon propagation in the interface region of the SiGe SL NWs is enhanced with SD. This unexpected phenomenon is explained by the increased interface area caused by the slipping of atomic planes in screw dislocated structures. The obtained results reveal that screw dislocations can result in a major suppression of thermal conductivity not only in homogeneous materials, but also in heterostructures with multiple interfaces. In combination with various other phonon suppressing methods [47-49], SD can reduce lattice thermal conductivity and boost thermoelectric performance.

\section{ACKNOWLEDGMENTS}

This work is supported by the Fundamental Research Funds for the Central Universities (A0920502051904-66). Y. $\mathrm{Ni}$ acknowledges the support of National Natural Science Foundation of China (NSFC) Grant No. 11774294, and the R\&D Program for International S\&T Cooperation and Exchanges of Sichuan province (Grant No. 2018HH0088).
[1] B. Sun, G. Haunschild, C. Polanco, J. Ju, L. Lindsay, G. Koblmuller, and Y. K. Koh, Nat. Mater. 18, 136 (2019).
[2] N. Zhang, P. Carrez, and R. Shahsavari, ACS Appl. Mater. Inter. 9, 1496 (2017). 
[3] R. L. Sproull, M. Moss, and H. Weinstock, J. Appl. Phys. 30, 334 (1959).

[4] P. G. Klemens, Solid State Physics, Vol. 7 (Academic Press, New York, 1958).

[5] P. G. Klemens, Proc. Phys. Soc. A. 68, 1113 (2002).

[6] Y. Ni, S. Xiong, S. Volz, and T. Dumitrică, Phys. Rev. Lett. 113, 124301 (2014).

[7] J. Al-Ghalith and T. Dumitrică, Screw-Dislocated Nanostructures (Springer International, New York, 2018).

[8] S. Xiong, J. Ma, S. Volz, and T. Dumitrică, Small 10, 1756 (2014).

[9] J. Alghalith, Y. Ni, and T. Dumitrică, Phys. Chem. Chem. Phys. 18, 9888 (2016).

[10] E. Akatyeva, L. Kou, I. Nikiforov, T. Frauenheim, and T. Dumitrică, ACS Nano. 6, 10042 (2012).

[11] I. Nikiforov, D. B. Zhang, and T. Dumitrică, J. Phys. Chem. Lett. 2, 2544 (2011).

[12] K. Termentzidis, M. Isaiev, A. Salnikova, I. Belabbas, D. Lacroix, and J. Kioseoglou, Phys. Chem. Chem. Phys. 20, 5159 (2018).

[13] M. J. Bierman, Y. K. A. Lau, A. V. Kvit, A. L. Schmitt, and S. Jin, Science 320, 1060 (2008).

[14] B. W. Jacobs, M. A. Crimp, K. McElroy, and V. M. Ayres, Nano Lett. 8, 4353 (2008).

[15] J. Zhu, H. Peng, A. F. Marshall, D. M. Barnett, W. D. Nix, and Y. Cui, Nat. Nano. 3, 477 (2008).

[16] S. A. Morin and S. Jin, Nano Lett. 10, 3459 (2010).

[17] F. Meng, S. A. Morin, A. Forticaux, and S. Jin, Acc. Chem. Res. 46, 1616 (2013).

[18] M. I. Bodnarchuk, L. Liang, F. Alice, N. Sigrid, R. F. Ismagilov, and D. V. Talapin, J. Am. Chem. Soc. 133, 8956 (2011).

[19] A. Akey, C. Lu, L. Yang, and I. P. Herman, Nano Lett. 10, 1517 (2010).

[20] H. Zhang, H. Han, S. Xiong, H. Wang, S. Volz, and Y. Ni, Appl. Phys. Lett. 111, 121907 (2017).

[21] E. S. Landry and A. J. H. Mcgaughey, J. Appl. Phys. 107, 013521 (2010).

[22] X. Li and R. Yang, Phys. Rev. B. 86, 054305 (2012).

[23] M. Hu and D. Poulikakos, Nano Lett. 12, 5487 (2012).

[24] G. Balasubramanian and I. K. Puri, Appl. Phys. Lett. 99, 013116 (2011).

[25] V. Samvedi and V. Tomar, Nanotechnology 20, 365701 (2009).

[26] X. Mu, L. Wang, X. Yang, P. Zhang, A. C. To, and T. Luo, Sci. Rep. 5, 16697 (2015).
[27] E. S. Landry and A. J. H. McGaughey, Phys. Rev. B. 80, 165304 (2009).

[28] Y. Chalopin, K. Esfarjani, A. Henry, S. Volz, and G. Chen, Phys. Rev. B. 85, 195302 (2012).

[29] Z. Tian, K. Esfarjani, and G. Chen, Phys. Rev. B. 86, 235304 (2012).

[30] R. Rurali, X. Cartoixa, and L. Colombo, Phys. Rev. B. 90, 041408(R) (2014).

[31] P. Chen, N. A. Katcho, J. P. Feser, W. Li, M. Glaser, O. G. Schmidt, D. G. Cahill, N. Mingo, and A. Rastelli, Phys. Rev. Lett. 111, 115901 (2013).

[32] B. Becker, P. K. Schelling, and S. R. Phillpot, J. Appl. Phys. 99, 123715 (2006)

[33] S. Plimpton, J. Comput. Phys. 117, 1 (1995).

[34] J. Tersoff, Phys. Rev. B 39, 5566 (1989).

[35] J. Tersoff, Phys. Rev. B 41, 3248 (1990).

[36] R. Kubo, M. Toda, and N. Hashitsume, Statistical Physics II (Springer, Berlin, 1985).

[37] See Supplemental Material at http://link.aps.org/supplemental/ 10.1103/PhysRevB.100.075432 for the thermal conductivity calculated from Stillinger-Weber interatomic potential, the running thermal conductivity for SiGe SL NW as a function of correlation time, and the influence of the NW cross-section size.

[38] K. Sääskilahti, J. Oksanen, J. Tulkki, and S. Volz, Phys. Rev. B. 90, 134312 (2014).

[39] K. Sääskilahti, J. Oksanen, S. Volz, and J. Tulkki, Phys. Rev. B 91, 115426 (2015).

[40] J. Wang and J. S. Wang, Appl. Phys. Lett. 88, 111909 (2006).

[41] T. Yamamoto, S. Konabe, J. Shiomi, and S. Maruyama, Appl. Phys. Exp. 2, 095003 (2013).

[42] H. Zhao and J. B. Freund, J Appl. Phys. 97, 024903 (2005).

[43] H. Bao, J. Chen, X. Gu, and B. Cao, ES Energy Environ. 1, 16 (2018).

[44] J. Garg and G. Chen, Phys. Rev. B. 87, 140302(R) (2013).

[45] G. Chen, Nanoscale Energy Transport and Conversion: A Parallel Treatment of Electrons, Molecules, Phonons, and Photons (Oxford University Press Inc, United States, 2005).

[46] S. Merabia and K. Termentzidis, Phys. Rev. B. 89, 054309 (2014).

[47] S. Xiong, K. Saaskilahti, Y. A. Kosevich, H. Han, D. Donadio, and S. Volz, Phys. Rev. Lett. 117, 025503 (2016).

[48] C. Shao, X. Yu, N. Yang, Y. Yue, and H. Bao, Nanoscale Microscale Thermophys. Eng. 21, 201 (2017).

[49] S. Xiong, D. Selli, S. Neogi, and D. Donadio, Phys. Rev. B 95, 180301(R) (2017). 